\title{
An extension of Jensen's discrete inequality to half convex functions
}

Vasile Cirtoaje ${ }^{*}$ and Alina Baiesu

* Correspondence: vcirtoaje@upgploiesti.ro

Department of Automatic Control and Computers, University of

Ploiesti, 100680 Ploiesti, Romania

\section{Abstract}

We extend the right and left convex function theorems to weighted Jensen's type inequalities, and then combine the new theorems in a single one applicable to a half convex function $f(u)$, defined on a real interval $\llbracket$ and convex for $u \leq s$ or $u \geq s$, where $s \in \mathbb{~}$. The obtained results are applied for proving some open relevant inequalities.

Keywords: weighted Jensen's discrete inequality, right convex function, left convex function, half convex function

\section{Introduction}

The right convex function theorem (RCF-Theorem) has the following statement (see [1-3]).

RCF-Theorem. Let $f(u)$ be a function defined on a real interval \ and convex for $u \geq s \in \mathbb{u}$. The inequality

$$
f\left(x_{1}\right)+f\left(x_{2}\right)+\cdots+f\left(x_{n}\right) \geq n f\left(\frac{x_{1}+x_{2}+\cdots+x_{n}}{n}\right)
$$

holds for all $x_{1}, x_{2}, \ldots, x_{n} \in \llbracket$ satisfying $x_{1}+x_{2}+\ldots+x_{n} \geq n$ s if and only if

$$
f(x)+(n-1) f(y) \geq n f(s)
$$

for all $x, y \in \square$ which satisfy $x \leq s \leq y$ and $x+(n-1) y=n s$.

Replacing $f(u)$ by $f(-u), s$ by $-s, x$ by $-x, y$ by $-y$, and each $x_{i}$ by $-x_{i}$ for $i=1,2, \ldots, n$, from RCF-Theorem we get the left convex function theorem (LCF-Theorem).

LCF-Theorem. Let $f(u)$ be a function defined on a real interval $\square$ and convex for

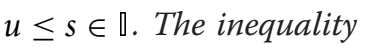

$$
f\left(x_{1}\right)+f\left(x_{2}\right)+\cdots+f\left(x_{n}\right) \geq n f\left(\frac{x_{1}+x_{2}+\cdots+x_{n}}{n}\right)
$$

holds for all $x_{1}, x_{2}, \ldots, x_{n} \in \llbracket$ satisfying $x_{1}+x_{2}+\ldots+x_{n} \leq n s$ if and only if

$$
f(x)+(n-1) f(y) \geq n f(s)
$$

for all $x, y \in \llbracket$ which satisfy $x \geq s \geq y$ and $x+(n-1) y=n s$.

Notice that from RCF- and LCF-Theorems, we get the following theorem, which we have called the half convex function theorem (HCF-Theorem). 
HCF-Theorem. Let $f(u)$ be a function defined on a real interval $\llbracket$ and convex for $u \leq s$

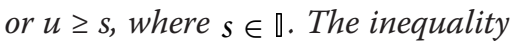

$$
f\left(x_{1}\right)+f\left(x_{2}\right)+\cdots+f\left(x_{n}\right) \geq n f\left(\frac{x_{1}+x_{2}+\cdots+x_{n}}{n}\right)
$$

holds for all $x_{1}, x_{2}, \ldots, x_{n} \in \llbracket$ satisfying $x_{1}+x_{2}+\ldots+x_{n}=n$ s if and only if

$$
f(x)+(n-1) f(y) \geq n f(s)
$$

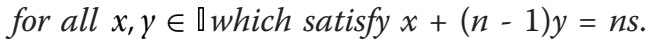

Applying RCF-, LCF-, and HCF-Theorems to the function $f(u)=g\left(e^{u}\right)$, and replacing $s$ by $\ln r, x$ by $\ln x, y$ by $\ln y$, and each $x_{i}$ by $\ln a_{i}$ for $i=1,2, \ldots, n$, we get the following corollaries, respectively.

RCF-Corollary. Let $g$ be a function defined on a positive interval $\square$ such that $f(u)=g$ $\left(e^{u}\right)$ is convex for $e^{u} \geq r \in \mathbb{\square}$. The inequality

$$
g\left(a_{1}\right)+g\left(a_{2}\right)+\cdots+g\left(a_{n}\right) \geq n g\left(\sqrt[n]{a_{1} a_{2} \ldots a_{n}}\right)
$$

holds for all $a_{1}, a_{2}, \ldots, a_{n} \in \llbracket$ satisfying $a_{1} a_{2} \ldots a_{n} \geq r^{n}$ if and only if

$$
g(a)+(n-1) g(b) \geq n g(r)
$$

for all $a, b \in \llbracket$ which satisfy $a \leq r \leq b$ and $a b^{n-1}=r^{n}$.

LCF-Corollary. Let $g$ be a function defined on a positive interval $\square$ such that $f(u)=g$ $\left(e^{u}\right)$ is convex for $e^{u} \leq r \in \mathbb{\square}$. The inequality

$$
g\left(a_{1}\right)+g\left(a_{2}\right)+\cdots+g\left(a_{n}\right) \geq n g\left(\sqrt[n]{a_{1} a_{2} \ldots a_{n}}\right)
$$

holds for all $a_{1}, a_{2}, \ldots, a_{n} \in \llbracket$ satisfying $a_{1} a_{2} \ldots a_{n} \leq r^{n}$ if and only if

$$
g(a)+(n-1) g(b) \geq n g(r)
$$

for all $a, b \in \llbracket$ which satisfy $a \geq r \geq b$ and $a b^{n-1}=r^{n}$.

HCF-Corollary. Let $g$ be a function defined on a positive interval $\llbracket$ such that $f(u)=g$ $\left(e^{u}\right)$ is convex for $e^{u} \leq r$ or $e^{u} \geq r$, where $r \in \mathbb{\square}$. The inequality

$$
g\left(a_{1}\right)+g\left(a_{2}\right)+\cdots+g\left(a_{n}\right) \geq n g(r)
$$

holds for all $a_{1}, a_{2}, \ldots, a_{n} \in \llbracket$ satisfying $a_{1} a_{2} \ldots a_{n}=r^{n}$ if and only if

$$
g(a)+(n-1) g(b) \geq n g(r)
$$

for all $a, b \in \mathbb{Q}$ which satisfy $a b^{n-1}=r^{n}$.

\section{Main results}

In order to extend RCF-, LCF-, and HCF-Theorems to weighted Jensen's type inequalities, we need the following lemma.

Lemma 2.1 Let $q_{1}, q_{2}$ and $r_{1}, r_{2}, \ldots, r_{m}$ be nonnegative real numbers such that

$$
r_{1}+r_{2}+\cdots+r_{m}=q_{1}+q_{2}
$$

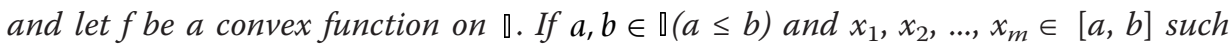
that 


$$
r_{1} x_{1}+r_{2} x_{2}+\cdots+r_{m} x_{m}=q_{1} a+q_{2} b
$$

then

$$
r_{1} f\left(x_{1}\right)+r_{2} f\left(x_{2}\right)+\cdots+r_{m} f\left(x_{m}\right) \leq q_{1} f(a)+q_{2} f(b) .
$$

The weighted right convex function theorem (WRCF-Theorem), weighted left convex function theorem (WLCF-Theorem), and weighted half convex function theorem (WHCF-Theorem) are the following.

WRCF-Theorem. Let $f(u)$ be a function defined on a real interval $\square$ and convex for $u \geq s \in \mathbb{\square}$, and let $p_{1}, p_{2}, \ldots, p_{n}$ be positive real numbers such that

$$
p=\min \left\{p_{1}, p_{2}, \ldots, p_{n}\right\}, \quad p_{1}+p_{2}+\cdots+p_{n}=1 \text {. }
$$

The inequality

$$
p_{1} f\left(x_{1}\right)+p_{2} f\left(x_{2}\right)+\cdots+p_{n} f\left(x_{n}\right) \geq f\left(p_{1} x_{1}+p_{2} x_{2}+\cdots+p_{n} x_{n}\right)
$$

holds for all $x_{1}, x_{2}, \ldots, x_{n} \in \llbracket$ satisfying $p_{1} x_{1}+p_{2} x_{2}+\ldots+p_{n} x_{n} \geq s$ if and only if

$$
p f(x)+(1-p) f(y) \geq f(s)
$$

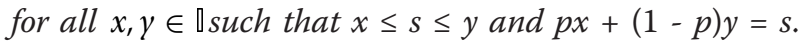

WLCF-Theorem. Let $f(u)$ be a function defined on a real interval $\rrbracket$ and convex for $u \leq s \in \mathbb{\square}$, and let $p_{1}, p_{2}, \ldots, p_{n}$ be positive real numbers such that

$$
p=\min \left\{p_{1}, p_{2}, \ldots, p_{n}\right\}, \quad p_{1}+p_{2}+\cdots+p_{n}=1 \text {. }
$$

The inequality

$$
p_{1} f\left(x_{1}\right)+p_{2} f\left(x_{2}\right)+\cdots+p_{n} f\left(x_{n}\right) \geq f\left(p_{1} x_{1}+p_{2} x_{2}+\cdots+p_{n} x_{n}\right)
$$

holds for all $x_{1}, x_{2}, \ldots, x_{n} \in \llbracket$ satisfying $p_{1} x_{1}+p_{2} x_{2}+\ldots+p_{n} x_{n} \leq s$ if and only if

$$
p f(x)+(1-p) f(y) \geq f(s)
$$

for all $x, y \in \llbracket$ such that $x \geq s \geq y$ and $p x+(1-p) y=s$.

WHCF-Theorem. Let $f(u)$ be a function defined on a real interval $\rrbracket$ and convex for $u$ $\leq s$ or $u \geq s$, where $s \in \mathbb{\mathbb { V }}$, and let $p_{1}, p_{2}, \ldots, p_{n}$ be positive real numbers such that

$$
p=\min \left\{p_{1}, p_{2}, \ldots, p_{n}\right\}, \quad p_{1}+p_{2}+\cdots+p_{n}=1 \text {. }
$$

The inequality

$$
p_{1} f\left(x_{1}\right)+p_{2} f\left(x_{2}\right)+\cdots+p_{n} f\left(x_{n}\right) \geq f\left(p_{1} x_{1}+p_{2} x_{2}+\cdots+p_{n} x_{n}\right)
$$

holds for all $x_{1}, x_{2}, \ldots, x_{n} \in \llbracket$ satisfying $p_{1} x_{1}+p_{2} x_{2}+\ldots+p_{n} x_{n}=s$ if and only if

$$
p f(x)+(1-p) f(y) \geq f(s)
$$

for all $x, y \in \llbracket$ such that $p x+(1-p) y=s$.

Notice that WLCF-Theorem can be obtained from WRCF-Theorem replacing $f(u)$ by $f(-u), s$ by $-s, x$ by $-x, y$ by $-y$, and each $x_{i}$ by $-x_{i}$ for $i=1,2, \ldots, n$.

On the other hand, applying WRCF-, WLCF-, and WHCF-Theorems to the function $f$ $(u)=g\left(e^{u}\right)$ and replacing $s$ by $1 \mathrm{n} r, x$ by $\ln x, y$ by $1 \mathrm{n} y$, and each $x_{i}$ by $\operatorname{In} a_{i}$ for $i=1,2, \ldots n$, we get the following corollaries, respectively. 
WRCF-Corollary Let $g$ be a function defined on a positive interval $\square$ such that $f(u)=$ $g\left(e^{u}\right)$ is convex for $\ln u \geq r \in \mathbb{\square}$, and let $p_{1}, p_{2}, \ldots, p_{n}$ be positive real numbers such that

$$
p=\min \left\{p_{1}, p_{2}, \ldots, p_{n}\right\}, \quad p_{1}+p_{2}+\cdots+p_{n}=1 .
$$

The inequality

$$
p_{1} g\left(a_{1}\right)+p_{2} g\left(a_{2}\right)+\cdots+p_{n} g\left(a_{n}\right) \geq g\left(a_{1}^{p_{1}} a_{2}^{p_{2}} \ldots a_{n}^{p_{n}}\right)
$$

holds for all $a_{1}, a_{2}, \ldots, a_{n} \in \llbracket$ satisfying $a_{1}^{p_{1}} a_{2}^{p_{2}} \ldots a_{n}^{p_{n}} \geq$ rif and only if

$$
p g(a)+(1-p) g(b) \geq g(r)
$$

for all $a, b \in \llbracket$ such that $a \leq r \leq b$ and $a^{p} b^{1-p}=r$.

WLCF-Corollary. Let $g$ be a function defined on a positive interval $\llbracket$ such that $f(u)=$ $g\left(e^{u}\right)$ is convex for $e^{u} \leq r \in \mathbb{Q}$, and let $p_{1}, p_{2}, \ldots, p_{n}$ be positive real numbers such that

$$
p=\min \left\{p_{1}, p_{2}, \ldots, p_{n}\right\}, \quad p_{1}+p_{2}+\cdots+p_{n}=1 .
$$

The inequality

$$
p_{1} g\left(a_{1}\right)+p_{2} g\left(a_{2}\right)+\cdots+p_{n} g\left(a_{n}\right) \geq g\left(a_{1}^{p_{1}} a_{2}^{p_{2}} \ldots a_{n}^{p_{n}}\right)
$$

holds for all $a_{1}, a_{2}, \ldots, a_{n} \in \llbracket$ satisfying $a_{1}^{p_{1}} a_{2}^{p_{2}} \ldots a_{n}^{p_{n}} \leq$ rif and only if

$$
p g(a)+(1-p) g(b) \geq g(r)
$$

for all $a, b \in \mathbb{\square}$ such that $a \geq r \geq b$ and $a^{p} b^{1-p}=r$.

WHCF-Corollary. Let $g$ be a function defined on a positive interval $\llbracket$ such that $f(u)$ $=g\left(e^{u}\right)$ is convex for $e^{u} \leq r$ or $e^{u} \geq r$, where $r \in \mathbb{\square}$, and let $p_{1}, p_{2}, \ldots, p_{n}$ be positive real numbers such that

$$
p=\min \left\{p_{1}, p_{2}, \ldots, p_{n}\right\}, \quad p_{1}+p_{2}+\cdots+p_{n}=1 .
$$

The inequality

$$
p_{1} g\left(a_{1}\right)+p_{2} g\left(a_{2}\right)+\cdots+p_{n} g\left(a_{n}\right) \geq g\left(a_{1}^{p_{1}} a_{2}^{p_{2}} \ldots a_{n}^{p_{n}}\right)
$$

holds for all $a_{1}, a_{2}, \ldots, a_{n} \in \llbracket$ satisfying $a_{1}^{p_{1}} a_{2}^{p_{2}} \ldots a_{n}^{p_{n}}=$ rif and only if

$$
p g(a)+(1-p) g(b) \geq g(r)
$$

for all $a, b \in \llbracket$ such that $a^{p} b^{1-p}=r$.

Remark 2.2. Let us denote

$$
g(u)=\frac{f(u)-f(s)}{u-s}, \quad h(x, y)=\frac{g(x)-g(y)}{x-y} .
$$

In some applications, it is useful to replace the hypothesis

$$
p f(x)+(1-p) f(y) \geq f(s)
$$

in WRCF-, WLCF-, and WHCF-Theorems by the equivalent condition: $h(x, y) \geq 0$ for all $x, y \in \mathbb{Q}$ such that $p x+(1-p) y=s$. 
This equivalence is true since

$$
\begin{aligned}
p f(x)+(1-p) f(y)-f(s) & =p[f(x)-f(s)]+(1-p)[f(y)-f(s)] \\
& =p(x-s) g(x)+(1-p)(y-s) g(y) \\
& =p(1-p)(x-\gamma)[g(x)-g(\gamma)] \\
& =p(1-p)(x-y)^{2} h(x, y) .
\end{aligned}
$$

Remark 2.3. The required inequalities in WRCF-, WLCF-, and WHCF-Theorems turn into equalities for $x_{1}=x_{2}=\ldots=x_{n}$. In addition, on the assumption that $p_{1}=\min$ $\left\{p_{1}, p_{2}, \ldots, p_{n}\right\}$, equality holds for $x_{1}=x$ and $x_{2}=\ldots=x_{n}=y$ if there exist $x, y \in \mathbb{\square}, x \neq$ $y$ such that $p x+(1-p) y=s$ and $p f(x)+(1-p) f(y)=f(s)$.

\section{Proof of Lemma 2.1}

Consider only the nontrivial case $a<b$. Since $x_{1}, x_{2}, \ldots, x_{m} \in[a, b]$ there exist $\lambda_{1}, \lambda_{2}, \ldots$, $\lambda_{m} \in[0,1]$ such that

$$
x_{i}=\lambda_{i} a+\left(1-\lambda_{i}\right) b, \quad i=1,2, \ldots, m .
$$

From

$$
\lambda_{i}=\frac{x_{i}-b}{a-b}, \quad i=1,2, \ldots, m
$$

we have

$$
\sum_{i=1}^{m} r_{i} \lambda_{i}=\frac{1}{a-b}\left(\sum_{i=1}^{m} r_{i} x_{i}-b \sum_{i=1}^{m} r_{i}\right)=\frac{1}{a-b}\left[q_{1} a+q_{2} b-b\left(q_{1}+q_{2}\right)\right]=q_{1} .
$$

Thus, according to Jensen's inequality, we get

$$
\begin{aligned}
\sum_{i=1}^{m} r_{i} f\left(x_{i}\right) & \leq \sum_{i=1}^{m} r_{i}\left[\lambda_{i} f(a)+\left(1-\lambda_{i}\right) f(b)\right] \\
& =[f(a)-f(b)] \sum_{i=1}^{m} r_{i} \lambda_{i}+f(b) \sum_{i=1}^{m} r_{i} \\
& =[f(a)-f(b)] q_{1}+f(b)\left(q_{1}+q_{2}\right) \\
& =q_{1} f(a)+q_{2} f(b) .
\end{aligned}
$$

\section{Proof of WRCF-Theorem}

Since the necessity is obvious, we prove further the sufficiency. Without loss of generality, assume that $x_{1} \leq x_{2} \leq \ldots \leq x_{n}$. If $x_{1} \geq s$, then the required inequality follows by Jensen's inequality for convex functions. Otherwise, since

$$
p_{1} x_{1}+p_{2} x_{2}+\cdots+p_{n} x_{n} \geq\left(p_{1}+p_{2}+\cdots+p_{n}\right) s,
$$

there exists $k \in\{1,2, \ldots, n-1\}$ such that

$$
x_{1} \leq \cdots \leq x_{k}<s \leq x_{k+1} \leq \cdots \leq x_{n} .
$$

Let us denote

$$
q=p_{1}+\cdots+p_{k}
$$


By Jensen's inequality, we have

$$
\left.\sum_{i=k+1}^{n} p_{i} f\left(x_{i}\right)\right) \geq\left(p_{k+1}+\cdots+p_{n}\right) f(z)=(1-q) f(z),
$$

where

$$
z=\frac{p_{k+1} x_{k+1}+\cdots+p_{n} x_{n}}{p_{k+1}+\cdots+p_{n}}, \quad z \geq s, \quad z \in \mathbb{U} .
$$

Thus, it suffices to prove that

$$
\sum_{i=1}^{k} p_{i} f\left(x_{i}\right)+(1-q) f(z) \geq f(S)
$$

where

$$
S=p_{1} x_{1}+p_{2} x_{2}+\cdots+p_{n} x_{n}=\frac{p_{1} x_{1}+p_{2} x_{2}+\cdots+p_{n} x_{n}}{p_{1}+p_{2}+\cdots+p_{n}}, \quad s \leq S \leq z .
$$

Let $y_{i}, i=1,2, \ldots, k$, defined by

$$
p x_{i}+(1-p) y_{i}=s .
$$

We will show that

$$
z \geq y_{1} \geq y_{2} \geq \cdots \geq y_{k}>s
$$

We have

$$
\begin{gathered}
y_{1} \geq y_{2} \geq \cdots \geq y_{k} \\
y_{k}-s=\frac{p\left(s-x_{k}\right)}{1-p}>0, \\
y_{1}=\frac{s-p x_{1}}{1-p} \leq \frac{S-p x_{1}}{1-p}=\frac{\left(p_{1}-p\right) x_{1}+p_{2} x_{2}+\cdots+p_{n} x_{n}}{\left(p_{1}-p\right)+p_{2}+\cdots+p_{n}} .
\end{gathered}
$$

Since $p_{1}-p=p_{1}-\min \left\{p_{1}, p_{2}, \ldots, p_{n}\right\} \geq 0$, we get

$$
\frac{\left(p_{1}-p\right) x_{1}+p_{2} x_{2}+\cdots+p_{n} x_{n}}{\left(p_{1}-p\right)+p_{2}+\cdots+p_{n}} \leq \frac{p_{2} x_{2}+\cdots+p_{n} x_{n}}{p_{2}+\cdots+p_{n}} \leq z
$$

and hence $y_{1} \leq z$. Now, from $z \geq y_{1} \geq y_{2} \geq \ldots \geq y k>s$, it follows that $y_{1}, y_{2}, \ldots, y_{k} \in \mathbb{\square}$. Then, by hypothesis, we have

$$
p f\left(x_{i}\right)+(1-p) f\left(y_{i}\right) \geq f(s)
$$

for $i=1,2, \ldots, k$. Summing all these inequalities multiplied by $p_{i} / p$, respectively, we get

$$
\sum_{i=1}^{k} p_{i} f\left(x_{i}\right)+\frac{1-p}{p} \sum_{i=1}^{k} p_{i} f\left(y_{i}\right) \geq \frac{q}{p} f(s)
$$

Therefore, to prove (36), it suffices to show that

$$
\frac{q}{p} f(s)+(1-q) f(z) \geq \frac{1-p}{p} \sum_{i=1}^{k} p_{i} f\left(y_{i}\right)+f(S) .
$$


Since $S \in[s, z], y_{i} \in(s, z]$ for $i=1,2, \ldots, k$,

$$
\begin{aligned}
& \frac{q}{p}+1-q=\frac{1-p}{p} \sum_{i=1}^{k} p_{i}+1, \\
& \frac{q}{p} s+(1-q) z=\frac{1-p}{p} \sum_{i=1}^{k} p_{i} y_{i}+S,
\end{aligned}
$$

(37) is a consequence of Lemma 2.1, where $m=k+1, a=s, b=z, q_{1}=q / p, q_{2}=1$ $q, r_{m}=1, x_{m}=S, r_{i}=(1-p) p_{i} / p$ and $x_{i}=y_{i}$ for $i=1,2, \ldots, k$.

\section{Applications}

Proposition 5.1. Let $a_{1}, a_{2}, \ldots, a_{n}(n \geq 3)$ be positive real numbers such that $a_{1} a_{2} \ldots a_{n}$ $=1$. If $p$ and $q$ are nonnegative real numbers such that $p+q \geq n-1$, then [4]

$$
\frac{1}{1+p a_{1}+q a_{1}^{2}}+\frac{1}{1+p a_{2}+q a_{2}^{2}}+\cdots+\frac{1}{1+p a_{n}+q a_{n}^{2}} \geq \frac{n}{1+p+q} .
$$

Proof. Write the desired inequality as

$$
g\left(a_{1}\right)+g\left(a_{2}\right)+\cdots+g\left(a_{n}\right) \geq n g(1),
$$

where

$$
g(t)=\frac{1}{1+p t+q t^{2}}, \quad t>0
$$

To prove this inequality, we apply HCF-Corollary for $r=1$. Let

$$
f(u)=g\left(e^{u}\right)=\frac{1}{1+p e^{u}+q e^{2 u}}, \quad u \in \mathbb{R} .
$$

Using the second derivative,

$$
f^{\prime \prime}(u)=\frac{e^{u}\left[4 q^{2} e^{3 u}+3 p q e^{2 u}+\left(p^{2}-4 q\right) e^{u}-p\right]}{\left(1+p e^{u}+q e^{2 u}\right)^{3}},
$$

we will show that $f(u)$ is convex for $e^{u} \geq r=1$. We need to show that

$$
4 q^{2} t^{3}+3 p q t^{2}+\left(p^{2}-4 q\right) t-p \geq 0
$$

for $t \geq 1$. Indeed,

$$
\begin{aligned}
4 q^{2} t^{3}+3 p q t^{2}+\left(p^{2}-4 q\right) t-p & \geq\left(4 q^{2}+3 p q+p^{2}-4 q-p\right) t \\
& =\left[(p+2 q)(p+q-2)+2 q^{2}+p\right] t>0
\end{aligned}
$$

because $p+q \geq n-1 \geq 2$.

By HCF-Corollary, it suffices to prove that $g(a)+(n-1) g(b) \geq n g(1)$ for all $a, b>0$ such that $a b^{n-1}=1$. We write this inequality as

$$
\frac{b^{2 n-2}}{b^{2 n-2}+p b^{n-1}+q}+\frac{n-1}{1+p b+q b^{2}} \geq \frac{n}{1+p+q} \text {. }
$$

Applying the Cauchy-Schwarz inequality, it suffices to prove that

$$
\frac{\left(b^{n-1}+n-1\right)^{2}}{\left(b^{2 n-2}+p b^{n-1}+q\right)+(n-1)\left(1+p b+q b^{2}\right)} \geq \frac{n}{1+p+q}
$$


which is equivalent to

$$
p B+q C \geq A,
$$

where

$$
\begin{gathered}
A=(n-1)\left(b^{n-1}-1\right)^{2} \geq 0 \\
B=\left(b^{n-1}-1\right)^{2}+n E=\frac{A}{n-1}+n E \\
C=\left(b^{n-1}-1\right)^{2}+n F=\frac{A}{n-1}+n F
\end{gathered}
$$

with

$$
E=b^{n-1}+n-2-(n-1) b, \quad F=2 b^{n-1}+n-3-(n-1) b^{2} .
$$

By the AM-GM inequality applied to $n-1$ positive numbers, we have $E \geq 0$ and $F \geq$ 0 for $n \geq 3$. Since $A \geq 0$ and $p+q \geq n-1$, we have

$$
p B+q C-A \geq p B+q C-\frac{(p+q) A}{n-1}=n(p E+q F) \geq 0 .
$$

Equality holds for $a_{1}=a_{2}=\ldots=a_{n}=1$.

Remark 5.2. For $p+q=n-1$ and $n \geq 3$, by Proposition 5.1 we get the following beautiful inequality

$$
\frac{1}{1+p a_{1}+q a_{1}^{2}}+\frac{1}{1+p a_{2}+q a_{2}^{2}}+\cdots+\frac{1}{1+p a_{n}+q a_{n}^{2}} \geq 1 .
$$

If $p=n-1$ and $q=0$, then we get the well-known inequality

$$
\frac{1}{1+(n-1) a_{1}}+\frac{1}{1+(n-1) a_{2}}+\cdots+\frac{1}{1+(n-1) a_{n}} \geq 1 .
$$

Remark 5.3. For $p=q=\frac{1}{r}, 0<r \leq \frac{2}{n-1}$ and $n \geq 3$, by Proposition 5.1 we obtain the inequality

$$
\frac{1}{r+a_{1}+a_{1}^{2}}+\frac{1}{r+a_{2}+a_{2}^{2}}+\cdots+\frac{1}{r+a_{n}+a_{n}^{2}} \geq \frac{n}{r+2} .
$$

In addition, for $r=\frac{2}{n-1}, n \geq 3$, we get

$$
\sum_{i=1}^{n} \frac{1}{2+(n-1)\left(a_{i}+a_{i}^{2}\right)} \geq \frac{1}{2}
$$

Remark 5.4. For $p=2 r, q=r^{2}, r \geq \sqrt{n}-1$ and $n \geq 3$, by Proposition 5.1 we obtain

$$
\frac{1}{\left(1+r a_{1}\right)^{2}}+\frac{1}{\left(1+r a_{2}\right)^{2}}+\cdots+\frac{1}{\left(1+r a_{n}\right)^{2}} \geq \frac{n}{(1+r)^{2}} .
$$

Proposition 5.5. Let $a_{1}, a_{2}, \ldots, a_{n}(n \geq 4)$ be positive real numbers such that $a_{1} a_{2} \ldots$ $a_{n}=1$. If $p, q, r$ are nonnegative real numbers such that $p+q+r \geq n-1$, then [4] 


$$
\sum_{i=1}^{n} \frac{1}{1+p a_{i}+q a_{i}^{2}+r a_{i}^{3}} \geq \frac{n}{1+p+q+r}
$$

Proof. Write the required inequality as

$$
g\left(a_{1}\right)+g\left(a_{2}\right)+\cdots+g\left(a_{n}\right) \geq n g(1)
$$

where

$$
g(t)=\frac{1}{1+p t+q t^{2}+r t^{3}}, \quad t>0,
$$

and apply HCF-Corollary to $g(t)$ for $r=1$. Let

$$
f(u)=g\left(e^{u}\right)=\frac{1}{1+p e^{u}+q e^{2 u}+r e^{3 u}},
$$

defined on $\mathbb{R}$. For $n \geq 4$, which implies $p+q+r \geq 3$, we claim that $f$ is convex for $e^{u} \geq 1$. Since

$$
f^{\prime \prime}(u)=\frac{t\left[9 r^{2} t^{5}+11 q r t^{4}+\left(2 p r+4 q^{2}\right) t^{3}+(3 p q-9 r) t^{2}+\left(p^{2}-4 q\right) t-p\right]}{\left(1+p t+q t^{2}+r t^{3}\right)^{3}},
$$

where $t=e^{u} \geq 1$, we need to show that

$$
9 r^{2} t^{5}+11 q r t^{4}+\left(2 p r+4 q^{2}\right) t^{3}+(3 p q-9 r) t^{2}+\left(p^{2}-4 q\right) t-p \geq 0
$$

Since

$$
9 r^{2} t^{5}+11 q r t^{4}+\left(2 p r+4 q^{2}\right) t^{3}-p \geq\left(9 r^{2}+11 q r+2 p r+4 q^{2}\right) t^{3}-p t,
$$

it suffices to show that

$$
\left(9 r^{2}+11 q r+2 p r+4 q^{2}\right) t^{2}+(3 p q-9 r) t+p^{2}-p-4 q \geq 0 .
$$

Using the inequality $t^{2} \geq 2 t-1$, we still have to prove that $A t+B \geq 0$, where

$$
\begin{gathered}
A=18 r^{2}+22 q r+4 p r+8 q^{2}+3 p q-9 r \\
B=-9 r^{2}-11 q r-2 p r-4 q^{2}+p^{2}-p-4 q .
\end{gathered}
$$

Since $p+q+r \geq 3$, we have

$$
\begin{aligned}
A & \geq 18 r^{2}+22 q r+4 p r+8 q^{2}+3 p q-3 r(p+q+r) \\
& =15 r^{2}+19 q r+p r+8 q^{2}+3 p q \geq 0 .
\end{aligned}
$$

Therefore,

$$
\begin{aligned}
A t & +B \geq A+B=p^{2}+4 q^{2}+9 r^{2}+3 p q+11 q r+2 p r-(p+4 q+9 r) \\
& \geq p^{2}+4 q^{2}+9 r^{2}+3 p q+11 q r+2 p r-\frac{(p+4 q+9 r)(p+q+r)}{3} \\
& =\frac{2(p-r)^{2}+9 q^{2}+16 r^{2}+4 p q+20 q r}{3}>0 .
\end{aligned}
$$

According to HCF-Corollary, it suffices to prove that $g(a)+(n-1) g(b) \geq n g(1)$ for all $a, b>0$ such that $a b^{n-1}=1$. We write this inequality as 


$$
\frac{b^{3 n-3}}{b^{3 n-3}+p b^{2 n-2}+q b^{n-1}+r}+\frac{n-1}{1+p b+q b^{2}+r b^{3}} \geq \frac{n}{1+p+q+r}
$$

or

$$
p^{2} A_{11}+q^{2} A_{22}+r^{2} A_{33}+p q A_{12}+q r A_{23}+r p A_{31} \geq A p+B q+C r,
$$

where

$$
\begin{aligned}
& A_{11}=b^{2 n-2}\left(b^{n}-n b+n-1\right), \\
& A_{22}=b^{n-1}\left(b^{2 n}-n b^{2}+n-1\right), \\
& A_{33}=b^{3 n}-n b^{3}+n-1, \\
& A_{12}=b^{3 n-1}+b^{3 n-2}+(n-1)\left(b^{2 n-2}+b^{n-1}\right)-n\left(b^{2 n}+b^{n}\right), \\
& A_{23}=b^{3 n}+b^{3 n-1}+(n-1)\left(b^{n-1}+1\right)-n\left(b^{n+2}+b^{2}\right), \\
& A_{31}=b^{3 n}+b^{3 n-2}+(n-1)\left(b^{2 n-2}+1\right)-n\left(b^{2 n+1}+b\right), \\
& A=b^{2 n-2}\left[(n-1) b^{n}-n b^{n-1}+1\right], \\
& B=b^{n-1}\left[(n-1) b^{2 n}-n b^{2 n-2}+1\right], \\
& C=(n-1) b^{3 n}-n b^{3 n-3}+1 .
\end{aligned}
$$

Since $A, B, C \geq 0$ (from the AM-GM inequality applied to $n$ positive numbers) and $p$ $+q+r \geq n-1$, it suffices to show that

$$
\begin{gathered}
(n-1)\left(p^{2} A_{11}+q^{2} A_{22}+r^{2} A_{33}+p q A_{12}+q r A_{23}+r p A_{31}\right) \geq \\
\geq(p+q+r)(A p+B q+C r),
\end{gathered}
$$

which is equivalent to

$$
p^{2} B_{11}+q^{2} B_{22}+r^{2} B_{33}+p q B_{12}+q r B_{23}+r p B_{31} \geq 0,
$$

where

$$
\begin{aligned}
B_{11} & =(n-1) A_{11}-A=n b^{2 n-2}\left[b^{n-1}-(n-1) b+n-2\right], \\
B_{22} & =(n-1) A_{22}-B=n b^{n-1}\left[b^{2 n-2}-(n-1) b^{2}+n-2\right], \\
B_{33} & =(n-1) A_{33}-C=n\left[b^{3 n-3}-(n-1) b^{3}+n-2\right] \\
B_{12} & =(n-1) A_{12}-A-B \\
& =n b^{n-1}\left[2 b^{2 n-2}-(n-1) b^{n+1}+(n-2) b^{n-1}-(n-1) b+n-2\right] \\
& =n b^{2 n-2}\left[2 b^{n-1}-(n-1) b^{2}+n-3\right]+n b^{n-1}\left[b^{n-1}-(n-1) b+n-2\right], \\
B_{23} & =(n-1) A_{23}-B-C \\
& =n\left[2 b^{3 n-3}-(n-1) b^{n+2}+(n-2) b^{n-1}-(n-1) b^{2}+n-2\right], \\
B_{31} & =(n-1) A_{31}-C-A \\
& =n\left[2 b^{3 n-3}-(n-1) b^{2 n+1}+(n-2) b^{2 n-2}-(n-1) b+n-2\right] .
\end{aligned}
$$


We see that $B_{11}, B_{22}, B_{33}, B_{12} \geq 0$ (by the AM-GM inequality applied to $n-1$ positive numbers). Also, we have

$$
\begin{aligned}
\frac{B_{23}}{n}= & b^{n-1}\left[3 b^{n-1}-(n-1) b^{3}+n-4\right]+2 b^{n-1}\left(b^{n-1}-1\right)^{2} \\
& +b^{2 n-2}-(n-1) b^{2}+n-2 \geq 0,
\end{aligned}
$$

since

$$
3 b^{n-1}-(n-1) b^{3}+n-4 \geq 0, \quad b^{2 n-2}-(n-1) b^{2}+n-2 \geq 0
$$

(by the AM-GM inequality applied to $n-1$ positive numbers). Using the inequality $b^{n-1}-(n-1) b+n-2 \geq 0$,we get $B_{31} \geq D$, where

$$
D=n b^{n-1}\left[2 b^{2 n-2}-(n-1) b^{n+2}+(n-2) b^{n-1}-1\right] .
$$

To prove (38), it suffices to show that $p^{2} B_{11}+r^{2} B_{33}+p r D \geq 0$. This is true if $4 B_{11} B_{33} \geq D^{2}$; that is,

$$
\begin{aligned}
4\left[b^{n-1}-(n-1) b+n-2\right]\left[b^{3 n-3}-(n-1) b^{3}+n-2\right] \geq \\
\geq\left[2 b^{2 n-2}-(n-1) b^{n+2}+(n-2) b^{n-1}-1\right]^{2} .
\end{aligned}
$$

In the case $n=4$, (39) becomes in succession

$$
\begin{gathered}
4\left(b^{3}-3 b+2\right)\left(b^{9}-3 b^{3}+2\right) \geq\left(b^{6}-2 b^{3}+1\right)^{2} \\
4(b-1)^{2}(b+2)\left(b^{3}-1\right)^{2}\left(b^{3}+2\right) \geq\left(b^{3}-1\right)^{4} \\
(b-1)^{2}\left(b^{3}-1\right)^{2}\left(3 b^{4}+5 b^{3}-3 b^{2}+6 b+15\right) \geq 0 .
\end{gathered}
$$

Clearly, the last inequality is true. The inequality (39) also holds for $n \geq 5$, but we leave this to the reader to prove. Equality occurs for $a_{1}=a_{2}=\ldots=a_{n}=1$.

Remark 5.6. For $n=4$ and $p+q+r=3$, by Proposition 5.5 we get the following beautiful inequality

$$
\sum_{i=1}^{4} \frac{1}{1+p a_{i}+q a_{i}^{2}+r a_{i}^{3}} \geq 1 .
$$

In addition, for $p=q=r=1$, we get the known inequality ([2])

$$
\sum_{i=1}^{4} \frac{1}{1+a_{i}+a_{i}^{2}+a_{i}^{3}} \geq 1 .
$$

Conjecture 5.7. Let $a_{1}, a_{2}, \ldots, a_{n}$ be positive real numbers such that $a_{1} a_{2} \ldots a_{n}=1$, and let $k_{1}, k_{2}, \ldots, k_{m}$ be nonnegative real numbers such that $k_{1}+k_{2}+\ldots+k_{m} \geq n-1$. If $m \leq n-1$, then

$$
\sum_{i=1}^{n} \frac{1}{1+k_{1} a_{i}+k_{2} a_{i}^{2}+\cdots+k_{m} a_{i}^{m}} \geq \frac{n}{1+k_{1}+k_{2}+\cdots+k_{m}} .
$$

Remark 5.8. For $m=n-1$ and $k_{1}=k_{2}=\ldots=k_{m}=1$, (40) turns into the known beautiful inequality ([2]) 


$$
\sum_{i=1}^{n} \frac{1}{1+a_{i}+a_{i}^{2}+\cdots+a_{i}^{n-1}} \geq 1
$$

Remark 5.9. For $k_{1}=\left(\begin{array}{c}m \\ 1\end{array}\right) r, k_{2}=\left(\begin{array}{c}m \\ 2\end{array}\right) r^{2}, \ldots, k_{m}=\left(\begin{array}{c}m \\ m\end{array}\right) r^{m}$, (40) turns into the known inequality $[1,2]$

$$
\sum_{i=1}^{n} \frac{1}{\left(1+r a_{i}\right)^{m}} \geq \frac{n}{(1+r)^{m}}
$$

which holds for $1 \leq m \leq n-1$ and $r \geq \sqrt[m]{n}-1$.

Proposition 5.10. If $x_{1}, x_{2}, \ldots, x_{n}$ are nonnegative real numbers such that

$$
x_{1}+2 x_{2}+\cdots+n x_{n}=\frac{n(n+1)}{2},
$$

then

$$
\begin{aligned}
& (n-1)(n+2)\left[x_{1}^{3}+2 x_{2}^{3}+\cdots+n x_{n}^{3}-\frac{n(n+1)}{2}\right] \geq \\
& \geq 2\left(n^{2}+n-1\right)\left[x_{1}^{2}+2 x_{2}^{2}+\cdots+n x_{n}^{2}-\frac{n(n+1)}{2}\right] .
\end{aligned}
$$

Proof. Since the inequality is trivial for $n=1$, consider further that $n \geq 2$. Write the inequality as

$$
p_{1} f\left(x_{1}\right)+p_{2} f\left(x_{2}\right)+\cdots+p_{n} f\left(x_{n}\right) \geq f\left(p_{1} x_{1}+p_{2} x_{2}+\cdots+p_{n} x_{n}\right),
$$

where

$$
\begin{gathered}
p_{i}=\frac{2 i}{n(n+1)}, \quad i=1,2, \ldots, n, \\
f(u)=(n-1)(n+2) u^{3}-2\left(n^{2}+n-1\right) u^{2}, \quad u \geq 0 .
\end{gathered}
$$

The function $f(u)$ is convex for $u \geq s=1$, since

$$
\begin{aligned}
f^{\prime \prime}(u) & =6(n-1)(n+2) u-4\left(n^{2}+n-1\right) \\
& \geq 6(n-1)(n+2)-4\left(n^{2}+n-1\right)=2\left(n^{2}+n-4\right)>0
\end{aligned}
$$

for $u \geq 1$. According to WHCF-Theorem and Remark 2.2, it suffices to prove that $h$ $(x, y) \geq 0$ for all $x, y \geq 0$ such that $p x+(1-p) y=1$, where

$$
p=\min \left\{p_{1}, p_{2}, \ldots, p_{n}\right\}=\frac{2}{n(n+1)} .
$$

We have

$$
\begin{aligned}
& g(u)=\frac{f(u)-f(1)}{u-1}=(n-1)(n+2)\left(u^{2}+u+1\right)-2\left(n^{2}+n-1\right)(u+1), \\
& h(x, y)=\frac{g(x)-g(y)}{x-y}=(n-1)(n+2)(x+y+1)-2\left(n^{2}+n-1\right) .
\end{aligned}
$$

From $p x+(1-p) y=1$, we get

$$
\begin{aligned}
x+y & =x+\frac{1-p x}{1-p}=\frac{1+(1-2 p) x}{1-p} \\
& =\frac{n(n+1)+\left(n^{2}+n-4\right) x}{(n-1)(n+2)} \geq \frac{n(n+1)}{(n-1)(n+2)},
\end{aligned}
$$


and hence

$$
h(x, y) \geq(n-1)(n+2)\left[\frac{n(n+1)}{(n-1)(n+2)}+1\right]-2\left(n^{2}+n-1\right)=0 .
$$

This completes the proof. Equality holds for $a_{1}=a_{2}=\ldots a_{n}=1$, and also for $a_{1}=0$ and $a_{2}=\cdots=a_{n}=\frac{n(n+1)}{(n-1)(n+2)}$.

\section{Acknowledgements}

The authors are grateful to the referees for their useful comments.

\section{Authors' contributions}

VC conceived and proved the main results and their applications. AB performed numerical verification for all applications and prepared the first manuscript. Both authors read and approved the final manuscript.

\section{Competing interests}

The authors declare that they have no competing interests.

Received: 17 April 2011 Accepted: 31 October 2011 Published: 31 October 2011

\section{References}

1. Cirtoaje, V: A generalization of Jensen's inequality. Gazeta Matematica Seria A. 2, 124-138 (2005)

2. Cirtoaje, V: Algebraic Inequalities-Old and New Methods. GIL Publishing House, Zalau. (2006)

3. Tetiva, M: A new proof for the right convex function theorem. Gazeta Matematica Seria A. 2, 126-133 (2006)

4. Art of Problem Solving.http://www.artofproblemsolving.com/Forum/viewtopic.php?t=223266

doi:10.1186/1029-242X-2011-101

Cite this article as: Cirtoaje and Baiesu: An extension of Jensen's discrete inequality to half convex functions.

Journal of Inequalities and Applications 2011 2011:101.

\section{Submit your manuscript to a SpringerOpen ${ }^{\circ}$} journal and benefit from:

- Convenient online submission

Rigorous peer review

- Immediate publication on acceptance

- Open access: articles freely available online

- High visibility within the field

- Retaining the copyright to your article

Submit your next manuscript at $\gg$ springeropen.com 